\title{
Positron Emission Mammography (PEM): A Promising Technique for Detecting Breast Cancer
}

\author{
C J Thompson ${ }^{+}, \mathrm{K} \mathrm{Murthy}^{+}, \mathrm{Y}$ Picard $^{+}$, I N Weinberg ${ }^{* \#}$, R Mako* \\ ${ }^{+}$Montreal Neurological Institute, 3801 University St \\ McGill University, Montreal, Canada \\ ${ }^{*}$ F M Technologies, Fairfax Va, \\ ${ }^{* \#}$ Johns Hopkins Hospital, Baltimore Md.
}

\section{ABSTRACT}

We are developing a high specificity technique for detecting the increased metabolic rate of breast tumours. The glucose analog FDG is known to concentrate in breast tumours rendering them easily detectable in conventional PET scans. Since PET is a relatively expensive imaging technique it has not been used routinely in the detection of breast cancer. Positron emission mammography (PEM) will provide a highly efficient, high spatial resolution, and low cost positron imaging system whose metabolic images are co-registered with conventional mammography. Coincidences between two BGO blocks cut into $2 \times 2 \mathrm{~mm}$ squares coupled to two $7.5 \mathrm{~cm}$ square imaging PMTs are detected and back-projected to form realtime multiple plane images. The design is about 20 times more sensitive than a conventional multi-slice PET body scanner, so much less radio-pharmaceutical can be used, reducing the patient dose and cost per scan. Prototype detectors have been made and extensive measurements done. The device is expected to have an in-plane spatial resolution about $2 \mathrm{~mm}$ FWHM. Besides the application as a secondary screening tool the device may be beneficial in measuring a tumour's response to radio-therapy or chemo-therapy, as well as aiding the surgeon in optimizing the removal of malignant tissue.

\section{INTRODUCTION}

Current mammographic methods are unreliable in differentiating benign from malignant breast tumours, particularly in clinically occult lesions. Screening mammography has increased the number of Stage I $(<2 \mathrm{~cm})$ cancers found through early detection [1]. Early detection of cancer leads to a reduction in mortality, as tumour size correlates with the probability of metastasis at the time of diagnosis [1]. Unfortunately, the specificity of mammography is limited for nonpalpable cancers. As a result 70 to $90 \%$ of excisional biopsies performed on non-palpable tumours are found to be benign [2]. Unnecessary biopsies are economically and psychologically burdensome, and may also cause scarring which renders interpretation of subsequent mammographic follow-up more difficult. The above considerations highlight the need for a highly specific imaging modality as an alternative to biopsy for non-palpable breast tumours.

There is also a need for a cancer detection technique that is more sensitive than mammography. Nine percent of breast cancers detected by physical examination in the Breast Cancer
Detection Demonstration Project were not detectable with mammography. The sensitivity of mammography is particularly limited in women with dense breasts or multiple diffuse microcalcifications, or who have had prior diagnostic interventions (i.e., biopsy, subtotal mastectomy, or radiotherapy) [3].

- Improved detection of multi-focality would allow better determination as to which patients are candidates for breast conserving surgery. Multi-focal disease in patients with nonpalpable cancers is common (44-60\%). In many patients with multi-focal disease, mammography often shows one focus [4]. - Improved description of tumour margin prior to surgery would aid the surgeon in the decision about the volume and region of tissue to be resected. Better assessment as to whether residual tumour is present after surgery could reduce the incidence of inadequate local resection, which is believed to lead to local recurrence rate of approximately ten percent [5].

PET has been used to detect breast cancer[6,7,8,9], but is not generally employed because of the cost and availability when compared to mammography. A prototype PEM scanner is presently being built at the Montreal Neurological Institute. We present its design concepts, and Monte Carlo simulations of both normal and what we think will be "typical" pathological images from the prototype instrument.

\section{MATERIALS AND METHODS}

\section{A. Detector Configuration}

The PEM detectors are designed to fit into a conventional mammography unit. One fits between the $\mathrm{X}$-ray tube and the upper compression plate, the second fits inside the magnification cone which is used in magnification mammography. Figure 1 shows the light-tight box which houses one detector mounted in a Philips Mammo Diagnost-UC.

\section{Photo-multipliers}

The shape and size of the detectors is determined from the choice of Hamamatsu R3941-05 position sensitive photomultipliers (PS-PMTs). These have front surface area of $7.5 \mathrm{x}$ $7.5 \mathrm{~cm}$ and a useful field of view of about $6.5 \times 5.5 \mathrm{~cm}$. This is about the same area as a CCD mammography system. The positioning is done by reading out the signal from 18 anode wires in the $\mathrm{X}$ direction and 17 in the $\mathrm{Y}$ direction. The pitch of these wires is $3.75 \mathrm{~mm}$. The "05" version of this PMT has both resistor chains and amplifiers to provide readouts of the $X_{A}, Y_{A}, X_{B}$, and $Y_{B}$, signals from both ends of both chains 
mounted on its base. It also has an AC coupled signal lead from the last dynode to provide a fast signal for timing.

\section{Crystal Configuration}

Crystal arrays are mounted on the Hamamatsu R3941-05 PS-PMTs. We have chosen to cover the whole face of the PMT with BGO crystal even though the present read-out technique prevents identification of crystals outside the anode mesh area. These PS-PMTS have been used in miniature gamma cameras by Yasillo[10], and Watanabe[11]. We are working on a technique to extend the field of view and improve the spatial resolution using a multi-channel sparse readout similar to that of Bird et al.[12], but adapted to our timing and data sampling scheme.

\section{Crystal Cutting}

We chose to make the detector from four BGO crystals whose dimensions are $36 \times 36 \times 20 \mathrm{~mm}$. These are purchased and then cut in a grid pattern using a multiple blade diamond saw. We use an Ultra Tec precision saw [Ultra Tec, Santa Ana, CA]. This has been modified to allow multiple blades to be rotated on the drive shaft. We designed a vice [Tipal Instruments, Montreal, Canada] which holds the crystal during cutting. During cutting, six blades cut slots $0.15 \mathrm{~mm}$ wide separated by $1.85 \mathrm{~mm}$. Three cutting passes are made on each face of the crystal. After all cuts are made in one direction, the crystal is rotated through $90^{\circ}$ to allow cuts to be made at right angles to the previous cuts. After the cuts on one surface are complete, the crystal block is inverted and the pattern is repeated on the bottom side. The cuts on the bottom are offset from those on the top by $1.0 \mathrm{~mm}[13]$, and the depth of cut is adjusted to ensure that equal numbers of gamma rays interact with both halves. This technique gives a sampling interval of $1.0 \mathrm{~mm}$ even though the crystals are almost twice this width. By filling the gaps with opaque light reflecting material, we can channel the light in the crystal elements rather than allowing it to spread over the PMT surface[14].

\section{B. Data Acquisition System}

\section{Signal and Coincidence Processing}

The PS-PMTs have a signal connection to the last dynode which provides a fast trigger point from which the time of each event can be determined. A buffer amplifier has been designed to amplify this signal for input to a constant fraction discriminator (CFD). The PS-PMT has buffer amplifiers connected to each end of a resistor ladder. The anode wires are connected to nodes of the ladder network. Five metre long cables take the five analog signals $\left(X_{A}, X_{B}, Y_{A}, Y_{B}\right.$, and $\left.D_{12}\right)$ from each PMT separately and connect them to the signal processing module (SPM). The SPM produces scaled $\left(X_{A}\right.$ $\left.X_{B}\right),\left(Y_{A}-Y_{B}\right)$ and $\left(X_{A}+X_{B}+Y_{A}+Y_{B}\right)$ signals which are input to three channels of the ADC. The dynode signal is further amplified and input to a CFD. This produces a NIM timing pulse which is fed to one input of the coincidence circuit (CC). The CC, which can also register single events for calibration purposes, has an aperture of 1-10 nsec. A valid coincidence triggers a mono-stable circuit which fires at the peak of the energy and position signals. This strobes the ADC.

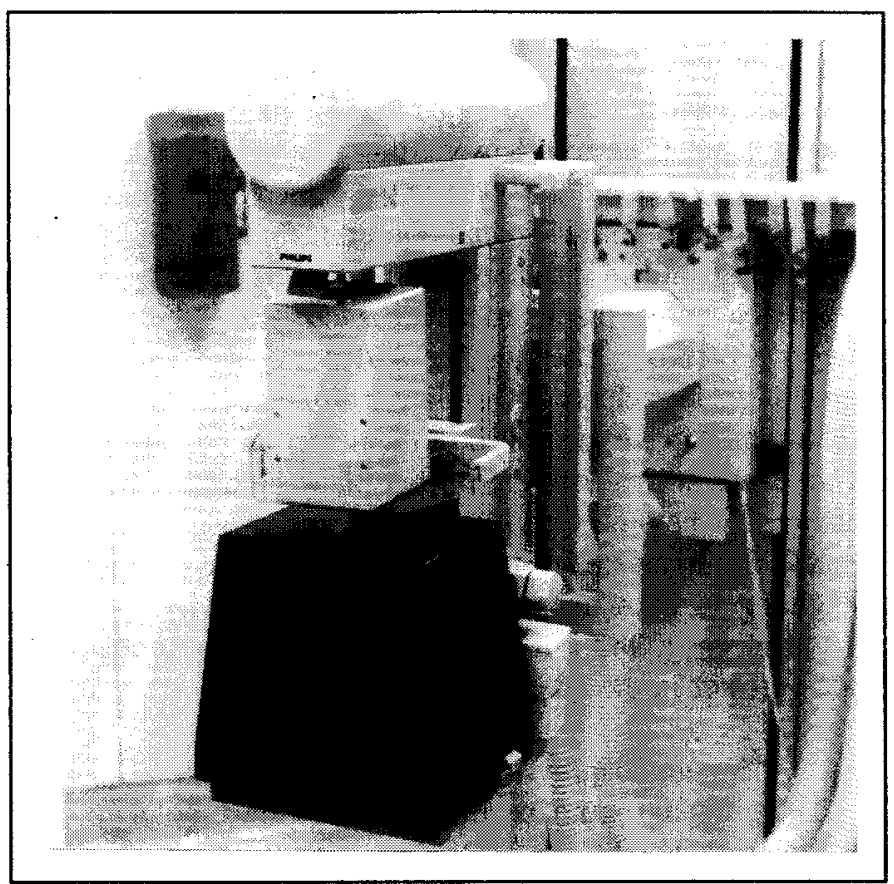

Figure 1 PEM detector module between the $X$-ray tube and upper compression plate of a Philips Mammo Diagnost-U C

The dynode amplifier, SPM and CC were designed for this project, and the SPM and CC are housed in dual NIM modules.

\section{ADC Triggering and Data Storage}

We use a Jorway [Jorway Corp., Westbury, NY] Aurora-14 CAMAC transient digitizer to acquire the two sets of energy and position signals. This unit is triggered only after a valid coincidence, and at the peak of the energy signal. The ADC has a $1 \mu$ sec dead time for a 12 bit conversion, and an internal memory of $128 \mathrm{k}$-words/channel. This memory is read out via a Jorway \#73A SCSI interface to the host computer, currently a VAXstation $4000 / 60$. The software polls the $\mathrm{ADC}$ at intervals related to the past count-rate and reads out the ADC memory in buffers which are multiples of 1024 events.

\section{Image Formation and Display}

Three interdependent modules acquire, transform and provide a real-time display of the PEM images. The first and second modules use a shared acquisition memory region and pass messages using VMS mailboxes, The second and third use a shared image memory region and also use mailboxes for synchronization.

\section{Acquisition, Compression and Storage Module}

This module takes the 12 bit ADC words from the input buffers and converts them to bytes, reformatting the matrix so that the six bytes associated with each event are in contiguous memory locations. This output buffer is saved as a list-mode file. This buffer is shared with image formation module.

\section{Image Computation Module}

This program, which is loaded by the user, first loads and then controls the other processes. This module processes the patient identification and file setup screens, and is controlled 
by the keyboard during acquisition. During acquisition, this module first identifies the $\mathrm{X}$ and $\mathrm{Y}$ coordinates of both rays as being associated with a unique crystal in either the top or bottom layer. It does this by finding the crystal address from a calibration table for that PMT-crystal combination. This process corrects for the spatial non-uniformity in the PMTs. If its energy is above the threshold, the event is back-projected into seven image planes each of which is a $128 \times 128$ long integer ( 32 bits). The number added to each image array depends on 1) each crystal's relative efficiency, 2) a weighting factor which is the reciprocal of the detection probability (DP) of an event at that location in that plane being detected. (see section $\mathbf{C ~} 4$ below). One location in each of the seven image planes is augmented when each coincidence occurs. The weighting function ensures that a uniform activity concentration produces a uniform image, even though the efficiency is much greater in the centre of the field of view.

\section{Image Display Module}

This module outputs to an $\mathrm{X}$-windows color display window set up as $14128 \times 128$ matrices, seven across and two down the screen. During acquisition one row is being updated each time the input buffer fills. The display contrast can be adjusted with the mouse during acquisition. The input can be re-directed from one row to another see how the image is changing with time. The appearance is similar to the simulation results in figure 4 .

\section{Weighting Backprojection for Image Uniformity}

The dimensions of the detectors are $2 X$ by $2 Y$ and their effective separation is $2 Z$. A positron annihilation at $x, y, z$ produces two gamma rays which make an angle $\alpha$ with the $\mathrm{z}$ axis projected on the $x, z$ plane, and $\beta$ with the $z$ axis projected on the $y, z$ plane. The detection probability,

$\operatorname{DP}(x, y, z, X, Y, Z)$ of detecting an event at $x, y, z$ is given by:

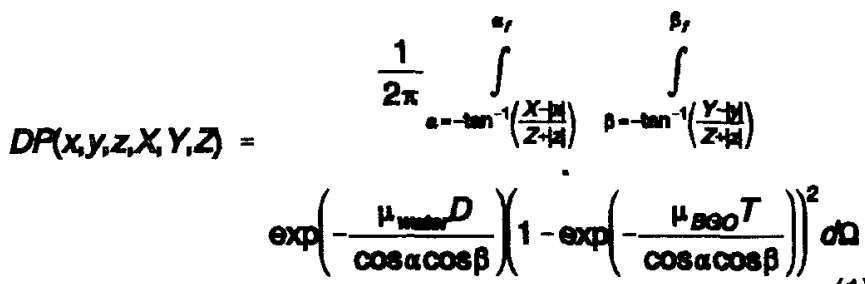

where $T$ is the thickness of the crystal, D is the thickness of the breast section and $\alpha_{\mathrm{f}}$ and $\beta_{\mathrm{f}}$ are defined as:

$$
\begin{aligned}
& \tan ^{-1} \frac{(x-|x|)}{(z-|z|)} \quad \mid \frac{z}{|x|}<\frac{z}{x} \\
& \alpha_{1}= \\
& \tan ^{-1} \frac{(x+|x|)}{(z+|z|)} \\
& \text { Othenmise }
\end{aligned}
$$

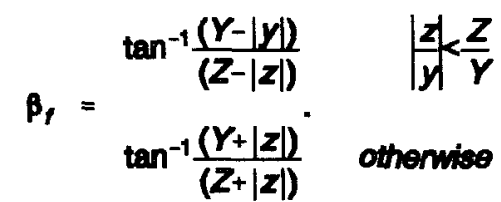

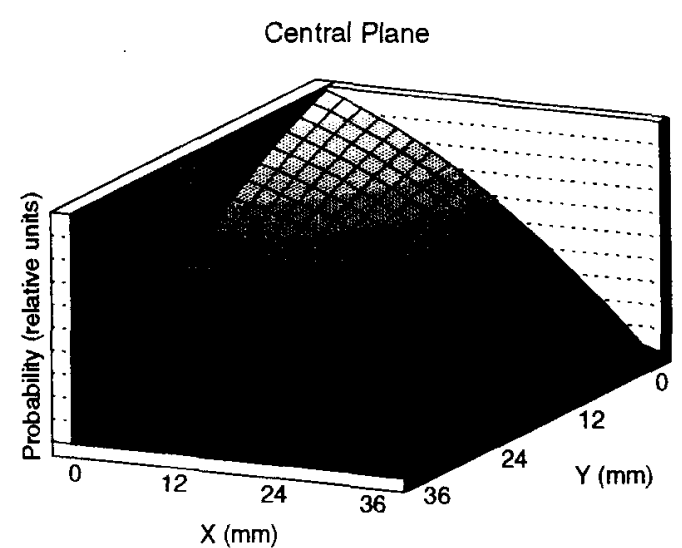

Figure 2 Detection probability function for points in a centra plane when the detector spacing is $50 \mathrm{~mm}$.

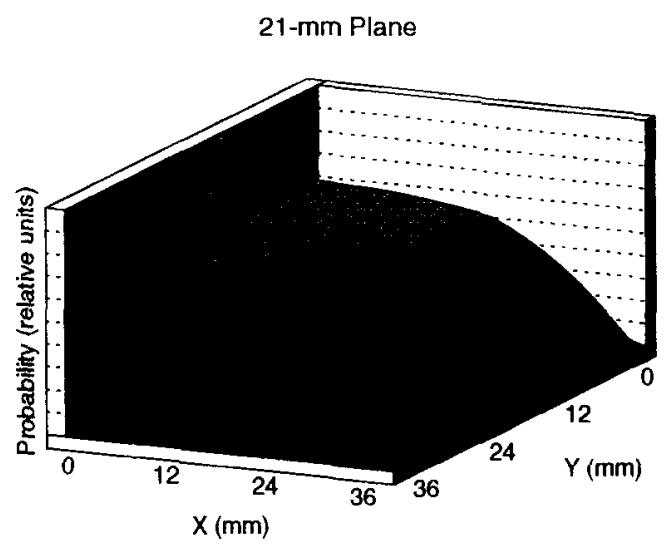

Figure 3 Detection probability function for points in a plane 21 $\mathrm{mm}$ from the centre when the detector spacing is $50 \mathrm{~mm}$.

The image is formed on seven planes by adding the reciprocal (RDP) of the detection probability $\mathbf{D P}(\mathbf{x}, \mathbf{y}, \mathbf{z}, \mathbf{X}, \mathbf{Y}, \mathbf{Z})$ given in equation 1 to the point $(x, y)$ at which the line joining the two coincident crystals intersects the $\mathrm{z}$ plane. Note that DP is independent of the angle of the individual gamma ray pair with respect to the detectors, and can thus be tabulated for look-up during display. The detectors are fixed size, and only their separation will change from study to study. The probability distribution for a detector separation of $50 \mathrm{~mm}$ is pictured in figure 2 for the central plane and in figure 3 for a plane 21 $\mathrm{mm}$ from the centre of the field.

\section{PEM Image Contrast}

The image contrast in PEM will be less than in conventional PET since no filtering is done to compensate for the backprojection onto several planes. If the breast is compressed to $\mathrm{D} \mathrm{cm}$ and a spherical lesion of radius $R$ has a true contrast 


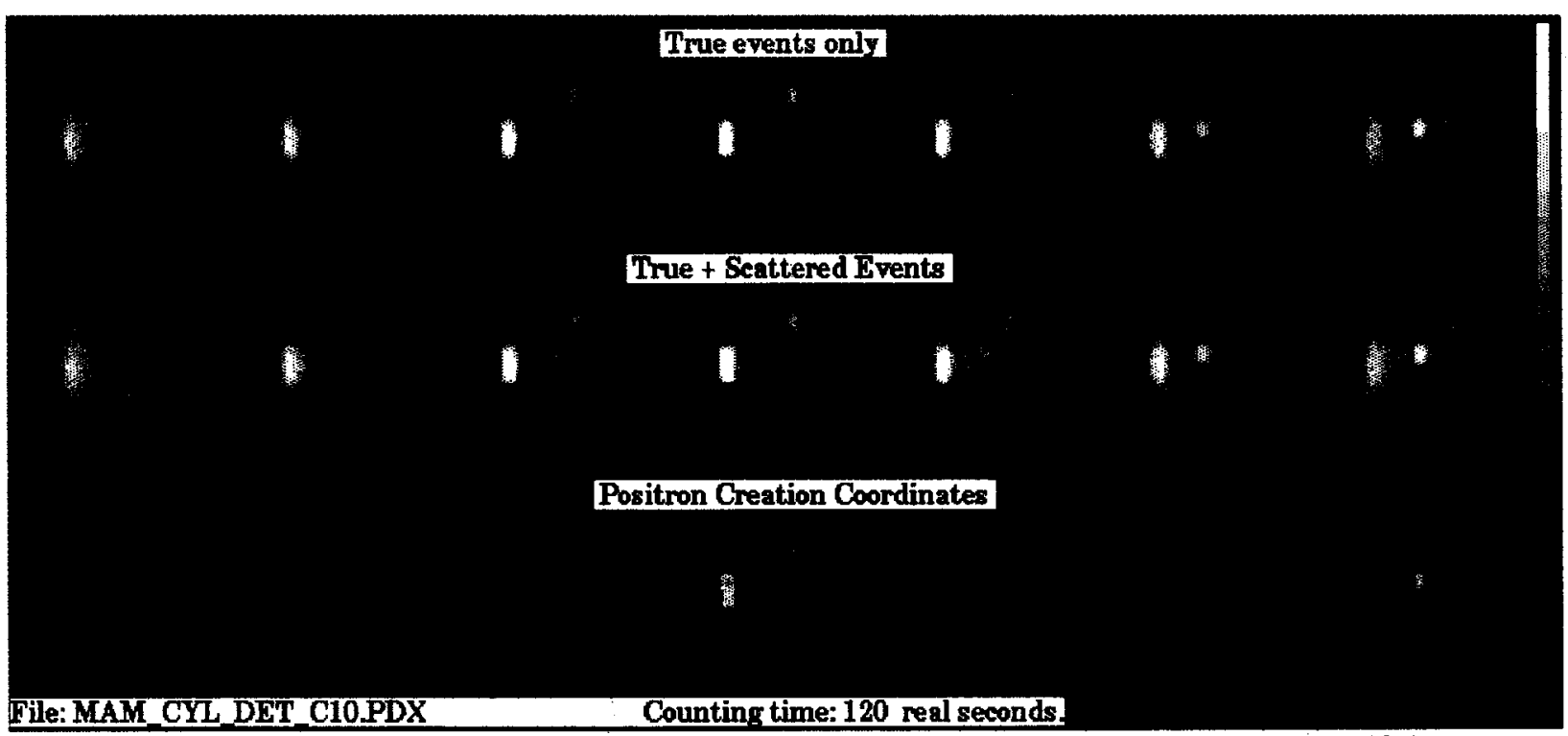

Figure 4 Two minute PEM scan with three lesions whose volume is from $0.3 \mathrm{ml}$ to $1.5 \mathrm{ml}$ with a $10: 1$ tumour to background ratio. The contrast is set to the minimum value.

of $\mathrm{C}_{\mathrm{TRUE}}: 1$, then assuming that $\mathrm{R}>2 * \mathrm{FWHM}$, the observed PEM contrast, $\mathrm{C}_{\mathrm{PEM}}$, will be:

$$
C_{\operatorname{mex}}=\frac{2 R\left(C_{n+\infty}-1\right)}{D}
$$

If the true contrast was 10:1 and the breast was compressed to $5 \mathrm{~cm}$ thick the observed PEM image contrast from a $1.0 \mathrm{~cm}$ diameter lesion would be $1.8: 1$. The lesion detectability will depend on the size of the lesion and the image noise.

\section{Simulated PEM Images}

Simulations of the noise-effective count-rate and efficiency have been presented elsewhere[15]. In order to test the image formation module, we modified the PETSIM[16] analysis module to provide images of the source and PEM output images. PETSIM retains the positron's spatial coordinates, and provides the centroid of all interactions within an active crystal detector. The $\mathrm{X}$ and $\mathrm{Y}$ coordinates in the plane nearest the annihilation are augmented by the $\mathrm{RDP}_{(X, Y, P)}$. These images are like auto-radiographs made by sandwiching a film between two sections of the breast each of which is half the slice thickness. Two other image sets are also produced. Into one of these sets both true and scattered events are back-projected and the $\mathrm{X}$ and $Y$ coordinates of the intersection point of the LOR and each plane are augmented by the RDP at that point. This is what we expect PEM images to look like. The final image set shows the result of back-projecting only the unscattered rays.

\section{Examples of Simulated PEM Scans}

We simulated a breast section compressed to $5 \mathrm{~cm}$ thick, as a box $7 \times 10 \mathrm{~cm}$ in the plane of the detector modules. The first simulation is for a uniform activity distribution corresponding to a "normal" PEM scan. The second scan has three "hot" cylindrical lesions which are 10 times the activity concentration of background. This tumour to background ratio is about what has been reported in previous PET studies in post-menopausal women[6]. The lesion range in size from 6 to $10 \mathrm{~mm}$ in diameter and together represent less than $0.5 \%$ of the total breast volume. In each case the imaging time corresponds to a 2 minute scan 40 minutes after the injection of $2 \mathrm{mCi}$ of FDG. The simulations include the effects of positron range for ${ }^{18} \mathrm{~F}$, the non-collinearity of the two gamma rays, and the effects of blurring due to multiple interactions within the detector block. The crystal density is $\mathbf{8 7 . 5 \%}$ of that of solid BGO due to the groves which separate the crystal segments.

\section{RESULTS}

The results of the simulations are shown in figure 4 . The abnormal scan has three cylindrical lesions, one on the left side in the central plane $1 \mathrm{~cm}$ in diameter and $3 \mathrm{~cm}$ long. A second, $0.8 \mathrm{~cm}$ diameter and $1 \mathrm{~cm}$ long, is in the lowest (right-most in the figure) plane. A third, $0.6 \mathrm{~cm}$ diameter and $1 \mathrm{~cm}$ long is in top-right quadrant of the central plane. The abnormal scan shows all three abnormalities in spite of the fact that these occupy less than $0.5 \%$ of the imaged volume. The contrast is much higher, and the events are better localized in the simulated "auto-radiographic image" (formed by mapping the positron creation coordinates onto the nearest plane) in the bottom row of figure 4 . This is to be expected since PEM image contrast is reduced according to equation 4 . These images are presented with the imaging window "wide open". If a narrower imaging window is used the perceived contrast improves substantially. The apparent contrast of each hot spot is sufficient to localize each lesion to a unique plane.

\section{v. DISCUSSION}

PEM is expected to be used in place of needle or surgical biopsy. Both FDG and C-11 Methionine have been shown to be highly specific markers for breast tumours. For PEM to replace biopsy as a diagnostic technique extensive clinical trials 
are required to evaluate its diagnostic specificity. At the present time all the detection electronics is functional, and we have to cut most of the crystals. Further work is required to evaluate the system resolution and efficiency before it is acceptable for human use.

The imaging performance obtained in the Monte Carlo simulations shows that diagnostic quality images should be obtained in reasonable imaging times. The two minute scans presented here are far longer than the $\mathrm{X}$-ray exposure in mammography, but the compression is much less too. The expected contrast will depend on the subject's age, as the fatty tissue remaining in post-menopausal women's breasts has a very low metabolic rate. This will increase the contrast substantially. However in younger patients, with dense breasts, the contrast will be reduced. The ability to digitally enhance the contrast by windowing the display, during acquisition should allow the imaging time to be optimized for patient comfort and diagnostic utility.

The device derives its in-plane spatial resolution from the ability to identify each of the crystals in the two layer BGO block. There will be some loss in spatial resolution due to Compton scattering in the block when the second ray deposits more energy than the first (as normally happens). The optical isolation of individual crystals by filling the saw cuts with white opaque material will prevent much of the blurring due to the natural light spread in the crystal which occurs in a conventional gamma camera. However until we are able to read out individual anode wires the spatial resolution will be limited as has been shown very well by Truman et al.[14]. He improved the spatial resolution from $1.3 \mathrm{~mm}$ FWHM to 0.9 $\mathrm{mm}$ using an array of $1.25 \times 1.25 \times 3.0 \mathrm{~mm}$ CsI crystals and $122 \mathrm{keV}$ gamma rays.

The axial resolution will depend on the extent to which the highly diagonal rays are used. The possibility of filtering the images to enhance the contrast along the inter-detector axis will be addressed after the basic system is functional. Conventional mammography has no axial resolution unless more than one view is made. This images in the middle row of figure 4 suggest that relatively high contrast objects should be correctly assigned to one plane. The axial resolution is not expected to be nearly as good as the in-plane resolution.

The most expensive items in the system are the PS-PMTs. The system uses only commercially available modules except the signal and coincidence processing, and these designs have been kept simple. The parts cost including the PS-PMTs, crystals, NIM and CAMAC electronics, and workstation is under US\$50,000.

\section{CONCLUSION}

We have presented at new technique which has very good potential for diagnosing breast cancer. The possibility of acquiring co-registered metabolic and radio-graphic images of the breast using a low cost technique should provide informa- tion comparable to that obtained only by biopsy at present. We are working to provide a system which has both a low radiation dose, and "cost per scan" by making the device as efficient and simple as possible.

\section{ACKNOWLEDGEMENTS}

We acknowledge support for our PET instrumentation research from NSERC grant \# OPG0036672 and MRC grant SP30. We would like to express their thanks to Dr. T Yamashita of Hamamatsu Photonics for arranging the loan of two imaging PMTs. We are very grateful for the many discussions with Dave Henricks of South Bay Technology and Joe Rubin of Ultra-Tec on the use of the diamond saw, and Tim Radway of Jorway on the programming of the data acquisition system. Mike Mazza, Bing Wang and Ray Clancy assisted with the construction and testing of the NIM modules.

\section{REFERENCES}

1. D.E. Henson, L.A. Ries: "Progress in Early Breast Cancer Detection", Cancer (1990) 65:2155-2158.

2. S.F. Lay, J.M. Crump, E.R. Frykberg, T.A. Goedde, E.M. Copeland: "Breast Biopsy - Changing Patterns During a Five-Year Period", The American Surgeon (1990) 56(2):79-85.

3. O.E. Niewig, E.E. Kim, W.H. Wong, W.F. Broussard, S.E. Singletary, G.N. Hortobagyi, R.S. Tilbury: "Positron Emission Tomography with Fluorine-18-Deoxyglucose in the Detection and Staging of Breast Cancer", Cancer (1993) 71:3920-5.

4. S.E. Harms, D.P. Flamig, K.L. Hesley, M.D. Meiches, R.A. Jensen, W.P. Evans, D.A. Savino, R.V. Wells: "MR Imaging of the Breast with Rotating Delivery of Excitation Off Resonance: Clinical Experience with Pathological Correlation", Radiology (1993) 187:493-501.

5. E.R. Fisher et al: "Pathological Findings from the National Surgical Adjuvant Breast Project (Protocol 6)", Cancer (1986) 57:1717-24.

6. Wahl R L, Cody R L, Hutchins G D, Mudgett E E: "Primary and metastatic breast carcinoma: initial clinical evaluation with PET with radio-labelled glucose analogue 2-[F-18]-flouro-2-deoxy-D-glucose." Radiology 197(3) pp 765-770 (1991)

7. Hunter G J, Choi N C, McLoud T C, and Fischman A J: "Lung tumour metastasis to breast detected by F-18-flourodeoxyglucose PET" J Nucl. Med. 34(9) pp 1571-1573 (1993)

8. Kim E E, Kim B T, Hynie T P, Podoloff D A, Wong W H, Yang D J, Tilbury R S, Hortobagyi, G, Singletary S, "Evaluation of preoperative chemotherapy in patients with loco-regionally advanced breast cancer using 18F-FDG PET", Journal of Nuclear Medicine, (Abstract), 33(5), pp 828, (1992).

9. Mintun, M A, M.J. Welch, B.A. Siegel, C.J. Mathias, J.W. Brodack, A.H. McGuire, J.A. Katzenellenbogen: "Breast Cancer: PET Imaging of Oestrogen Receptors." Radiology (1988) 169:45-48. 
10. Yasillo N J, Beck R N, Cooper M, "Design considerations for a single tube gamma camera" IEEE TNS 37:2 609-615 (1990)

11. Watanabe $M$, Uchida $H$, Okada $H$, Shimizu $K$ et al. "A high resolution PET for animal studies" IEEE Trans. Med Imag. 11:4 577-580 (1992)

12. Bird A J, He Z, Ramsden D. "Multi-channel readout of crossed-wire anode photomultipliers" Nucl Inst. and Meth. in Phys. Res. A 348 668-672 (1994)

13. Yamashita $T$, Watanabe $M$, Shimizu $K$, and Uchida $H$. "High resolution block detectors for PET". IEEE Trans Nucl Sci. NS-37 589-593 1990.

14. Truman A, Bird A J, Ramsden D, He Z, "Pixellated CsI(TI) arrays with position sensitive PMT readout." Nucl Inst. and Meth. in Phys. Res. A 353 375-378 1994

15. Thompson, CJ, Murthy K, Weinberg I, Mako F, "Feasibility Study for Positron Emission Mammography". Med. Phys. 21:4 pp 529-537 (1994)

16. Thompson CJ, Moreno-Cantu J, and Picard Y: "PETSIM: Monte Carlo Simulation of all Sensitivity and Resolution Parameters of Positron Imaging Systems". Phys. Med. Biol. 37:3 pp 731-749 (1992) 